\title{
Structure of the kinase domain of human RNA-dependent protein kinase with K296R mutation reveals a face-to-face dimer
}

\author{
LI FengZhi ${ }^{1,2 \dagger}$, LI SiWei $^{2,3 \dagger}$, WANG Zheng ${ }^{2,3}$, SHEN YueQuan ${ }^{2,3}$, ZHANG TongCun $^{1 *}$ \& \\ YANG Xue ${ }^{2,3 *}$ \\ ${ }^{1}$ Institute of Cell and Molecular Biology, Medical School, Wuhan University of Science and Technology, Wuhan 430065, China; \\ ${ }^{2}$ State Key Laboratory of Medicinal Chemical Biology, Nankai University, Tianjin 300071, China; \\ ${ }^{3}$ College of Life Sciences, Nankai University, Tianjin 300071, China
}

Received January 10, 2012; accepted May 30, 2012; published online October 29, 2012

\begin{abstract}
RNA-dependent protein kinase (PKR) is crucial for the innate immune response, cell growth, proliferation, signal transduction and apoptosis. The activation process of PKR has been studied for many years and is still under debate. To obtain new insight into the mechanism of PKR activation, we solved the crystal structure of a latent mutant of the PKR kinase domain (PKR-KD) in the apo form at a resolution of $2.9 \AA$. The overall structure of PKR-KD is similar to previously reported structures. Structural analysis revealed a classical back-to-back dimer and a newly defined face-to-face dimer. Analytical ultracentrifugation experiments, electrostatic surface maps and the model of PKR-KD in complex with the eIF2 $\alpha$ substrate all support that the face-to-face dimer is more reflective of PKR in solution. Our results provide new information on PKR dimerization and its activation mechanism.
\end{abstract}

PKR, dimerization, crystal structure, PKR activation

Citation: $\quad$ Li F Z, Li S W, Wang Z, et al. Structure of the kinase domain of human RNA-dependent protein kinase with K296R mutation reveals a face-to-face dimer. Chin Sci Bull, 2013, 58: 998-1002, doi: 10.1007/s11434-012-5461-z

Reversible phosphorylation of proteins, which is executed by kinases and phosphatases, constitutes a major form of signaling and is an essential mechanism of regulation in all living organisms. Ser/Thr protein kinases are one class of kinases and perform essential reactions in many cellular processes [1]. Double-stranded RNA-dependent protein kinase (PKR) is a Ser/Thr protein kinase that plays a key role in the innate immunity response to viral infection $[2,3]$. PKR exhibits low expression levels in normal cells. When intracellular or extracellular viral particles stimulate the body's innate immune response, cells produce interferons and simultaneously upregulate PKR. Double-stranded RNA (dsRNA) elements within the viral genome can induce activation of PKR [4]. Therefore, PKR can be considered as a cellular sensor of viral contamination. The activated PKR phosphorylates the eukaryotic initiation factor $2 \alpha(\mathrm{eIF} 2 \alpha)$ at

$\dagger$ These authors contributed equally to this work.

*Corresponding authors (email: zhangtongcun@wust.edu.cn; xueyangnk@yahoo.com)
Ser51, which then forms a sequestered inhibitory complex with eIF2B (guanine nucleotide exchange factor), causing inhibition of viral protein translation initiation $[5,6]$.

PKR consists of two functional domains (Figure 1(a)), the N-terminal RNA-binding domain and a C-terminal kinase domain, which are bridged by an 80-residue interdomain linker [7]. The N-terminal dsRNA-binding domain (referred as dsRBD) consists of two tandem motifs for RNA recognition. The dsRBD contains a conserved $\alpha \beta \beta \beta \alpha$ fold with two $\alpha$-helices that pack against a single face of a three-stranded antiparallel $\beta$-sheet. The C-terminal domain of PKR is a catalytic domain, which has a typical protein kinase structure; its $\mathrm{N}$-terminal lobe is mostly $\beta$-sheet, and its C-terminal lobe is predominantly helical [8].

The structure of PKR-KD in complex with eIF $2 \alpha$ revealed a back-to-back dimer with the $\mathrm{N}$-terminal lobes of the kinase domain as the interface. The latent PKR has been proposed to exist in a closed or autoinhibited conformation in which dsRBD interacts with the kinase domain. Upon 
(a)
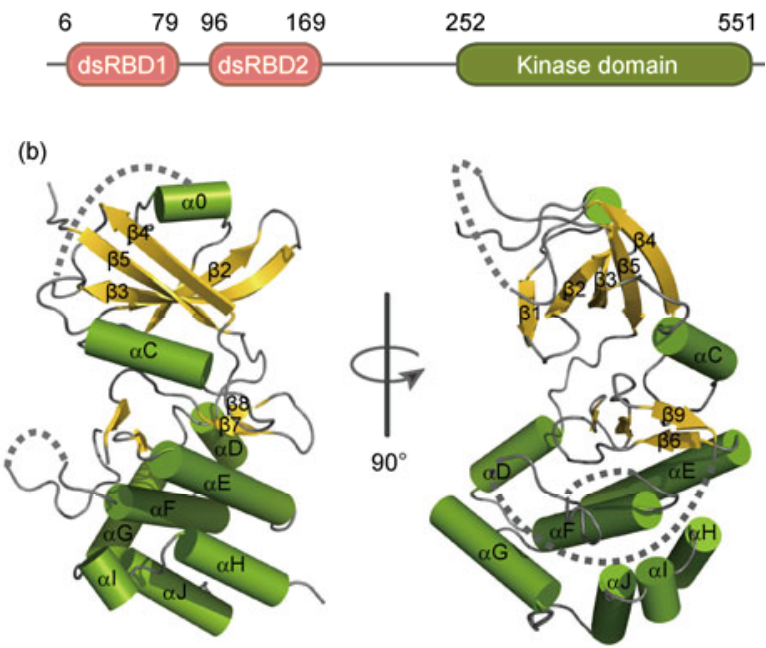

Figure 1 Overall structure of PKR-KD. (a) Schematic representation of the domain organization of human PKR. (b) Cartoon representation of PKRKD. Alpha-helices and beta-sheets are shown in green and gold, respectively.

binding dsRNA, the latent PKR is hypothesized to change its autoinhibited conformation, and the $\mathrm{N}$-lobe of the kinase domain forms a dimer that promotes autophosphorylation of the activation-segment residue Thr446, thereby leading to its efficient kinase activity [9-12]. Phosphorylation of Thr446 is critical for the catalytic efficiency of PKR and for specific substrate recognition $[13,14]$. The self-association of PKR is important for its kinase activation. In the absence of dsRNA, auto-phosphorylation of PKR can occur in high concentrations. Moreover, isolated PKR-KD can be activated by fusion with dimerization protein tag, indicating that dimerization of the kinase domain is sufficient to activate PKR in vitro [12]. However, the conformation of latent PKR in solution defined by small-angle X-ray scattering and small-angle neutron scattering is inconsistent with the autoinhibition model and showed that intrinsically disordered regions in PKR become ordered upon RNA binding, thereby promoting PKR dimerization [15].

To gain insight into the mechanism of PKR activation and substrate recognition, we determined the crystal structure of the catalytic domain of an inactive human PKR mutant (K296R). We propose that PKR-KD may form a face-to-face dimer to activate its downstream target.

\section{Materials and methods}

\subsection{Protein expression and purification}

The DNA fragment encoding PKR-KD (Homo sapiens) was amplified from a cDNA library of the HEK293 cell line and was cloned into the pET-28a vector (Novagen, USA), which has a C-terminal His6-tag. The K296R mutant was generated by the QuickChange (Stratagene, CA) method. The recombinant plasmid was transformed into Escherichia coli
BL21 CodonPlus (DE3) competent cells. The resulting cells were grown to $A_{600}=0.6-0.8$ in LB medium containing 30 $\mu \mathrm{g} / \mathrm{mL}$ kanamycin at $310 \mathrm{~K}$ and then induced by adding isopropyl $\beta$ - $D$-1-thiogalactopyranoside (IPTG) to a final concentration of $250 \mu \mathrm{mol} / \mathrm{L}$. The cells were harvested by centrifugation $16 \mathrm{~h}$ later. Next, the cells were resuspended in a buffer containing $500 \mathrm{mmol} / \mathrm{L} \mathrm{NaCl}, 1 \mathrm{mg} / \mathrm{mL}$ lysozyme and $20 \mathrm{mmol} / \mathrm{L}$ Tris- $\mathrm{HCl}(\mathrm{pH} 7.5)$, and then sonicated on ice for $3 \mathrm{~min}$ (with intervals of $1 \mathrm{~s}$ on and $3 \mathrm{~s}$ off). The supernatant obtained by centrifugation for $30 \mathrm{~min}$ at 20000 $\times g$ was used for protein purification.

The lysate was loaded directly onto a Ni-NTA column (QIAGEN, Valencia, CA), which was first equilibrated with 5 column volumes of buffer A $(500 \mathrm{mmol} / \mathrm{L} \mathrm{NaCl}, 20$ $\mathrm{mmol} / \mathrm{L}$ Tris-HCl, $\mathrm{pH}$ 7.5). Non-specifically bound proteins were removed by washing the column with buffer $\mathrm{C}$ (500 $\mathrm{mmol} / \mathrm{L} \mathrm{NaCl}, 20 \mathrm{mmol} / \mathrm{L}$ Tris- $\mathrm{HCl}$ and $150 \mathrm{mmol} / \mathrm{L}$ imidazole, $\mathrm{pH} 7.5$ ), and the recombinant $\mathrm{PKR}-\mathrm{KD}$ proteins were eluted with buffer D $(500 \mathrm{mmol} / \mathrm{L} \mathrm{NaCl}, 20 \mathrm{mmol} / \mathrm{L}$ Tris$\mathrm{HCl}$ and $500 \mathrm{mmol} / \mathrm{L}$ imidazole, $\mathrm{pH}$ 7.5). The proteins were then concentrated to $500 \mu \mathrm{L}$ with Centricon filters (Millipore, Belgium) and passed over a HiLoad Superdex 200 26/60 column (GE Healthcare Bio-Sciences, Co). The purified PKR-KD was eluted with a buffer containing 500 $\mathrm{mmol} / \mathrm{L} \mathrm{NaCl}, 20 \mathrm{mmol} / \mathrm{L}$ Tris-HCl, $1 \mathrm{mmol} / \mathrm{L}$ EDTA and $1 \mathrm{mmol} / \mathrm{L}$ DTT, pH 7.5. The PKR-KD mutant (PKR-KD Y404H-T446D), PKR-dsRBD2-KD K296R (residues 100551) and full-length PKR K296R were purified following the same protocol as used for PKR-KD K296R.

\subsection{Crystallization and data collection}

PKR-KD (30 mg/mL in $20 \mathrm{mmol} / \mathrm{L}$ Tris- $\mathrm{HCl}, 500 \mathrm{mmol} / \mathrm{L}$ $\mathrm{NaCl}, 1 \mathrm{mmol} / \mathrm{L}$ DTT and $1 \mathrm{mmol} / \mathrm{L}$ EDTA, pH 7.5) was crystallized using the sitting drop vapor diffusion method by equilibrating against a reservoir solution consisting of 100 $\mathrm{mmol} / \mathrm{L}$ MES, $200 \mathrm{mmol} / \mathrm{L}$ sodium fluoride and 20\% PEG$3350, \mathrm{pH}$ 6.5. Crystals were grown for one week at $277 \mathrm{~K}$ and flash-cooled with liquid nitrogen in a reservoir solution containing $25 \%$ glycerol. The data were collected on the BL17U1 beamline of the Shanghai Synchrotron Radiation Facility (SSRF) and then processed using the software HKL2000 [16]. The crystals diffracted to $2.9 \AA$ and belong to space group $\boldsymbol{P} 3_{1} 12$, with unit cell dimensions of $\boldsymbol{a}=\boldsymbol{b}=$ $95.4 \AA$ and $c=122.0 \AA$.

\subsection{Structure determination and refinement}

The initial phases were obtained by molecular replacement using the PKR-KD structure from the PKR-KD/eIF2alpha/ AMP-PNP complex (PDB code: 2A19) as a model. The program PHASER [17] located two molecules in one asymmetric unit. Model building and refinement were performed using COOT [18] and PHENIX [19]. The final structure has an $\boldsymbol{R}_{\text {crystal }}$ value of $27.9 \%$ and an $\boldsymbol{R}_{\text {free }}$ value of 
$30.5 \%$. The Ramachandran plot constructed by the program PROCHECK [20] showed that $72.3 \%$ of the residues are found in the most favored regions, $24.6 \%$ are in additionally allowed regions, $3.1 \%$ are in generously allowed regions and none of the residues are in disallowed regions. Detailed data collection and refinement statistics are summarized in Table 1. All figures were made by the program PyMol (DeLano Scientific LLC.).

\subsection{Analytical ultracentrifugation}

Sedimentation velocity (SV) experiments were performed in a Beckman/Coulter XL-I analytical ultracentrifuge using double-sector or 6-channel centerpieces and sapphirine windows. Before the experiments, the proteins were changed to the same buffer $(20 \mathrm{mmol} / \mathrm{L}$ Tris- $\mathrm{HCl}, 200 \mathrm{mmol} / \mathrm{L} \mathrm{NaCl}$ and $1 \mathrm{mmol} / \mathrm{L}$ EDTA, pH 7.5) using a HiLoad Superdex 200 26/60 column (GE Healthcare Bio- Sciences, Co). Experiments were conducted at $40000 \mathrm{r} / \mathrm{min}$. and $283 \mathrm{~K}$ using double-sector cells and were monitored at $280 \mathrm{~nm}$, where the absorbance of the solution ranged between 0.8 and 1.2. The data were analyzed using the program SEDFIT [21].

Table 1 Data collection and refinement statistics for PKR-KD

\begin{tabular}{|c|c|}
\hline Parameters & Results \\
\hline Space group & $P 3_{1} 12$ \\
\hline Unit cell $(\AA)$ & $a=b=95.4, c=122.0$ \\
\hline Wavelength (§) & 0.9795 \\
\hline Resolution range $(\AA)$ & $50-2.90(3.00-2.90)^{\mathrm{b})}$ \\
\hline No. of unique reflections & 14198 \\
\hline Redundancy & $10.9(7.6)^{\mathrm{b})}$ \\
\hline $\boldsymbol{R}_{\text {sym }}(\%)^{\text {a) }}$ & $6.8(78.6)^{b)}$ \\
\hline$I / \sigma$ & $36.4(1.8)^{\mathrm{b})}$ \\
\hline Completeness (\%) & $99.5(98.0)^{\mathrm{b})}$ \\
\hline \multicolumn{2}{|l|}{ Refinement } \\
\hline $\boldsymbol{R}_{\text {crystal }}(\%)^{\mathrm{c})}$ & 27.9 \\
\hline $\boldsymbol{R}_{\text {free }}(\%)^{\mathrm{d})}$ & 30.5 \\
\hline $\operatorname{RMSD}_{\text {bond }}(\AA)$ & 0.011 \\
\hline $\operatorname{RMSD}_{\text {angle }}(\AA)$ & 1.4 \\
\hline \multicolumn{2}{|l|}{ Number of } \\
\hline Protein atoms & 4066 \\
\hline Ligand atoms & 0 \\
\hline Solvent atoms & 24 \\
\hline \multicolumn{2}{|l|}{ Residues in (\%) } \\
\hline Most favored & 72.3 \\
\hline Additionally allowed & 24.6 \\
\hline Generously allowed & 3.1 \\
\hline Disallowed & 0 \\
\hline \multicolumn{2}{|l|}{ Average B factor $\left(\AA^{2}\right)$ of } \\
\hline Protein & 98.1 \\
\hline Solvent & 72.8 \\
\hline
\end{tabular}

a) $R_{\text {sym }}=\sum_{j}\left|\langle I\rangle-I_{j}\right| / \sum\langle I\rangle$; b) the highest resolution shell; c) $\boldsymbol{R}_{\text {cryst }}$ $=\sum_{\mathrm{hkl}} \mid F_{\mathrm{obs}}-F_{\text {calc }} / / \sum_{\mathrm{hkl}} F_{\mathrm{obs}} ;$ d) $\boldsymbol{R}_{\text {free }}$ was calculated in the same way as $\boldsymbol{R}_{\text {crystal }}$ but from a test set containing $5 \%$ of data excluded from the refinement calculation.

\subsection{Accession numbers}

The atomic coordinates and structure factors for the reported crystal structures were deposited in the Protein Data Bank with accession code 3 UIU for PKR-KD.

\section{Results and discussion}

\subsection{Overall structure of PKR-KD}

The structure of PKR-KD was solved by molecule replacement at a resolution of $2.9 \AA$. Two PKR-KD molecules were present in one asymmetric unit. The final model of molecules A and B consists of residues 254-336, 355-439, $451-460$ and $465-541$ of the total 510 residues.

The overall structure of PKR-KD adopts a typical kinase domain fold with an N-terminal lobe and a larger C-terminal lobe bridged by a short hinge (Figure 1(b)). The $\mathrm{N}$-lobe of the kinase domain consists of a 5-stranded antiparallel $\beta$ sheet $(\beta 1-\beta 5)$, one helix $\alpha 0$ on the top and another helix $\alpha \mathrm{C}$. Helix $\alpha \mathrm{C}$ contains the ATP-binding site (residue 273-281). The C-lobe is comprised of two pairs of antiparallel $\beta$ strands ( $\beta 7-\beta 8$ and $\beta 6-\beta 9)$ and eight $\alpha$ helices $(\alpha \mathrm{D}-$ $\alpha \mathrm{J})$. Helices $\alpha \mathrm{H}, \alpha \mathrm{I}$ and $\alpha \mathrm{J}$ are at the bottom of the whole structure. Helix $\alpha \mathrm{G}$ plays a critical role in substrate recognition.

\subsection{Dimeric structure of PKR-KD}

PKR-KD forms a back-to-back dimer (Figure 2(a)) by a 2-fold non-crystallographic symmetry axis and a face-toface dimer (Figure 2(b)) by a 2-fold crystallographic symmetry axis in the crystal structure. The superimposition of the PKR-KD/eIF2alpha/AMP-PNP complex molecule onto the molecule from our back-to-back and face-to-face models gave a RMSD value of $1.32 \AA$ for $243 \mathrm{C} \alpha$ atoms. The buried area calculated by AREAIMOL in the CCP4 suite [22] was $1844 \AA^{2}$ for the back-to-back dimer and $1640 \AA^{2}$ for the face-to-face dimer. The interactions are mediated by the two N-lobes within the back-to-back dimer, and no interaction was observed between the two C-lobes. By contrast, interactions occurred between both the $\mathrm{N}$ - and C-lobes in the face-to-face dimer.

To investigate the correlation of the dimers in the crystal and in solution, we took advantage of the analytical ultracentrifugation technique. The latent mutants (K296R) of full-length PKR, PKR-dsRBD2-KD and PKR-KD all exist as monomers in solution. However, the kinase domain of the mutant mimicking PKR phosphorylation (PKR-KD K296R-Y404H-T446D) is $90 \%$ dimeric and $10 \%$ monomeric in solution, which is distinctly different from the latent forms of PKR truncations (Figure 2(c)). These data indicate that PKR presumably exists as a monomer and the self-association is significantly increased after phosphorylation. In other words, phosphorylation promotes dimer formation. Whereas Thr446 is located on the dimeric interface 
(a)
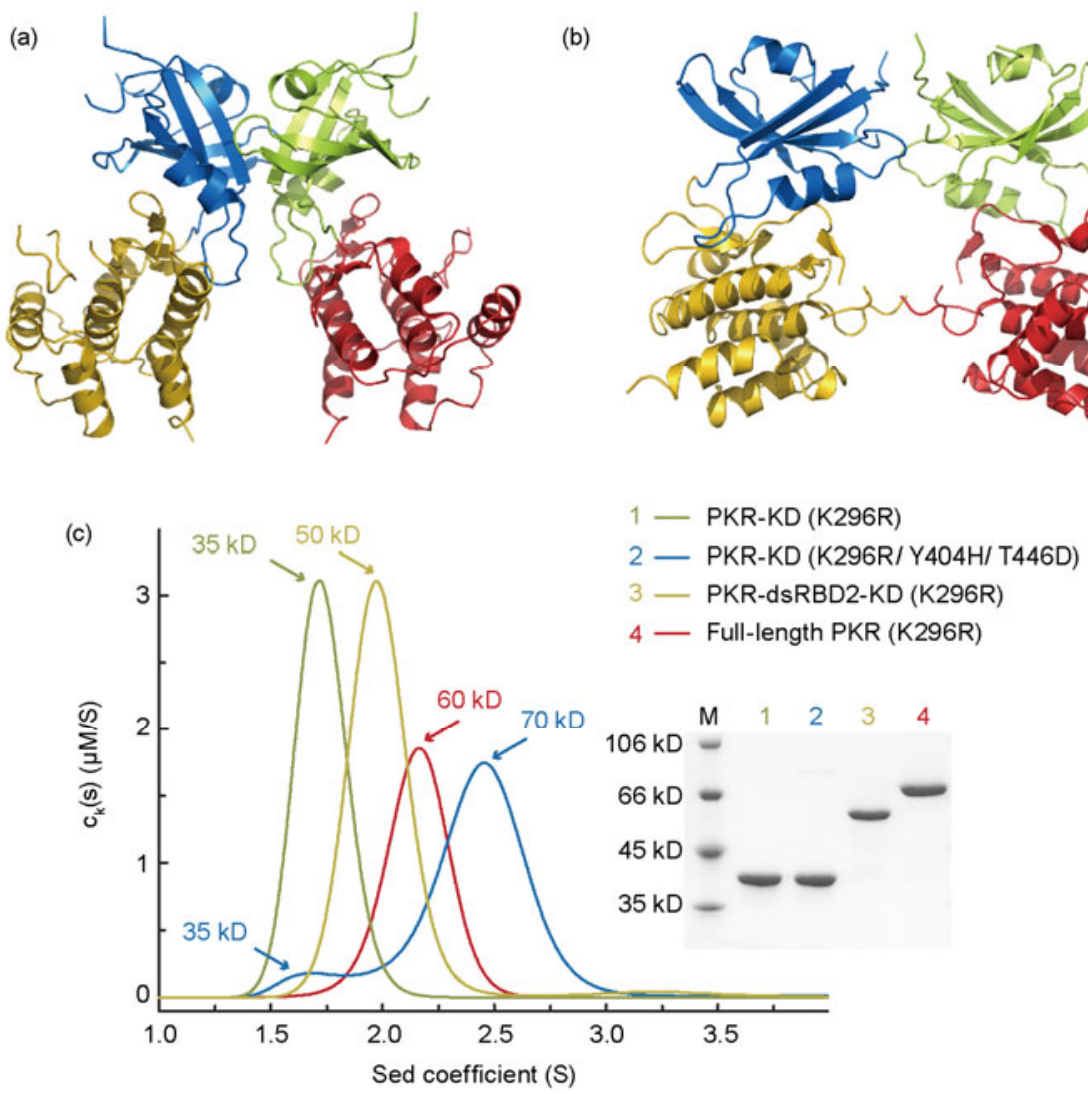

(b)

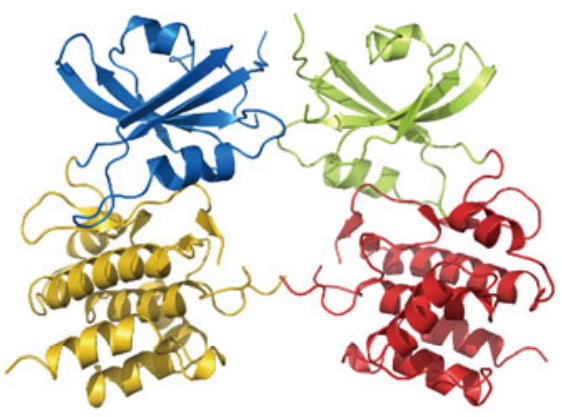

1 - PKR-KD (K296R)

2 - PKR-KD (K296R/ Y404H/ T446D)

3 - PKR-dsRBD2-KD (K296R)

Figure 2 Dimerization of PKR-KD. (a, b) Two forms of the PKR-KD dimer. (a) Back-to back dimer. (b) Face-to-face dimer. The N-lobe from chain A, the C-lobe from chain A, the N-lobe from chain B and the C-lobe from chain B are colored blue, yellow, green and red, respectively. (c) Sedimentation velocity (SV) measurements and SDS-PAGE of PKR mutants and truncations. M, Marker; 1, PKR-KD K296R; 2, PKR-KD K296R/Y404H/T446D; 3, PKRdsRBD2-KD K296R; 4, PKR-full length K296R.

of the face-to-face dimer, it is on the opposite face in the back-to-back dimer. Therefore, we speculate that the faceto-face dimer in the crystal may be more reflective of the PKR dimer in the solution.

We constructed an electrostatic surface map of PKR-KD (Figure 3). A positively charged area composed of Lys304, $\operatorname{Arg} 307$ and Arg413 was found at the center of the C-lobe. The negatively charged phosphorylation group may induce a charge-charge interaction to facilitate formation of the face-to-face dimer. We superimposed the previously determined structure of the PKR-KD/eIF $2 \alpha$ complex onto our face-to-face dimer (Figure 4 ). The eIF2 $\alpha$ molecule can interact with both monomers in the face-to-face dimer. The PKR dimer appears to be more favorable for the binding of eIF2 $\alpha$, in accordance with PKR dimerization being necessary to carry out its biological function in vivo.

\section{Conclusions}

In summary, we report the apo crystal structure of the K296R mutant of PKR kinase domain. Structural analysis revealed a previously unobserved face-to-face dimer, which seems to be more consistent with biochemical data.

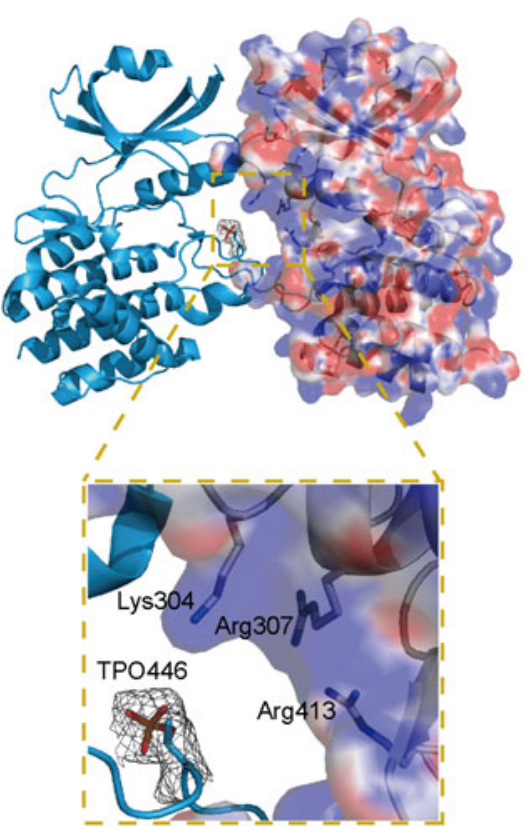

Figure 3 Electrostatic analysis of the face-to-face dimer interface. Chain B of the PKR complex (PDB code 2A19) is superimposed onto our face-toface model to represent the interaction of the phosphorylated Thr 446 and the positively charged cluster of the molecule that it faces. The $2 F_{\mathrm{O}}-\boldsymbol{F}_{\mathrm{c}}$ electron density map of phosphorylated Thr446 was contoured at $1.5 \sigma$ level. 


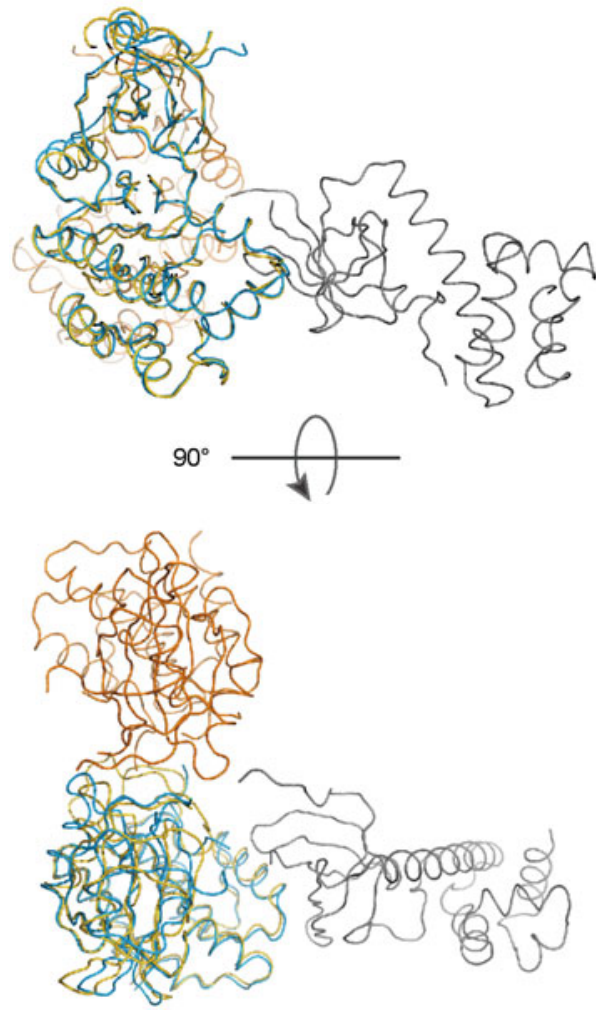

Figure 4 Superimposed face-to-face dimer and PKR-KD/elf2 $\alpha$ complex. Chain A and chain B in the face-to-face dimer are colored orange and cyan, respectively. PKR-KD and elf2 $\alpha$ in the complex (PDB 2A19) are colored gold and gray, respectively.

Although additional activity data will need to be collected to verify the face-to-face dimer, this model provides new information on the activation process of PKR.

We are grateful to the staff at the BL17U1 beamline of the Shanghai Synchrotron Radiation Facility for excellent technical assistance during data collection. This work was supported by the National Key Basic Program of China (2012CB917201 and 2009CB825504), the National Natural Science Foundation of China (31170684) and the Fundamental Research Funds for the Central Universities (65020241).

1 Shi Y. Serine/threonine phosphatases: Mechanism through structure. Cell, 2009, 139: 468-484

2 Nanduri S, Rahman F, Williams B R, et al. A dynamically tuned double-stranded RNA binding mechanism for the activation of antiviral kinase PKR. EMBO J, 2000, 19: 5567-5574

3 Taghavi N, Samuel C E. Protein kinase PKR catalytic activity is re- quired for the PKR-dependent activation of mitogen-activated protein kinases and amplification of interferon beta induction following virus infection. Virology, 2012, 427: 208-216

4 Pfaller C K, Li Z, George C X, et al. Protein kinase PKR and RNA adenosine deaminase ADAR1: New roles for old players as modulators of the interferon response. Curr Opin Immunol, 2011, 23: 573582

5 Dever T E. Gene-specific regulation by general translation factors. Cell, 2002, 108: 545-556

6 Dey M, Trieselmann B, Locke E G, et al. PKR and GCN2 kinases and guanine nucleotide exchange factor eukaryotic translation initiation factor 2B (eIF2B) recognize overlapping surfaces on eIF2alpha. Mol Cell Biol, 2005, 25: 3063-3075

7 Nallagatla S R, Toroney R, Bevilacqua P C. Regulation of innate immunity through RNA structure and the protein kinase PKR. Curr Opin Struct Biol, 2011, 21: 119-127

8 Nakamura T, Furuhashi M, Li P, et al. Double-stranded RNAdependent protein kinase links pathogen sensing with stress and metabolic homeostasis. Cell, 2010, 140: 338-348

9 Dar A C, Dever T E, Sicheri F. Higher-order substrate recognition of eIF2alpha by the RNA-dependent protein kinase PKR. Cell, 2005, 122: 887-900

10 Robertson H D, Mathews M B. The regulation of the protein kinase PKR by RNA. Biochimie, 1996, 78: 909-914

11 Cole J L. Activation of PKR: An open and shut case? Trends Biochem Sci, 2007, 32: 57-62

12 Dey M, Cao C, Dar A C, et al. Mechanistic link between PKR dimerization, autophosphorylation, and eIF2alpha substrate recognition. Cell, 2005, 122: 901-913

13 Romano P R, Garcia-Barrio M T, Zhang X, et al. Autophosphorylation in the activation loop is required for full kinase activity in vivo of human and yeast eukaryotic initiation factor 2alpha kinases PKR and GCN2. Mol Cell Biol, 1998, 18: 2282-2297

14 McKenna S A, Lindhout D A, Shimoike T, et al. Viral dsRNA inhibitors prevent self-association and autophosphorylation of PKR. J Mol Biol, 2007, 372: 103-113

15 VanOudenhove J, Anderson E, Krueger S, et al. Analysis of PKR structure by small-angle scattering. J Mol Biol, 2009, 387: 910-920

16 Otwinowski Z, Minor W. Processing of X-ray diffraction data collected in oscillation mode. Methods Enzymol, 1997, 276: 307-326

17 McCoy A J, Grosse-Kunstleve R W, Adams P D, et al. Phaser crystallographic software. J Appl Crystallogr, 2007, 40: 658-674

18 Emsley P, Lohkamp B, Scott W G, et al. Features and development of coot. Acta Crystallogr D Biol Crystallogr, 2010, 66: 486-501

19 Adams P D, Afonine P V, Bunkoczi G, et al. PHENIX: A comprehensive Python-based system for macromolecular structure solution. Acta Crystallogr D Biol Crystallogr, 2010, 66: 213-221

20 Laskowski R A, MacArthur M W, Moss D S, et al. PROCHECK: A program to check the stereochemical quality of protein structures. $\mathrm{J}$ Appl Crystallogr, 1993, 26: 283-291

21 Schuck P. Size-distribution analysis of macromolecules by sedimentation velocity ultracentrifugation and Lamm equation modeling. Biophys J, 2000, 78: 1606-1619

22 Winn M D, Ballard C C, Cowtan K D, et al. Overview of the CCP4 suite and current developments. Acta Crystallogr D Biol Crystallogr, 2011, 67: 235-242

Open Access This article is distributed under the terms of the Creative Commons Attribution License which permits any use, distribution, and reproduction in any medium, provided the original author(s) and source are credited. 